\title{
Rectal mucosal dysplasia in Crohn's disease
}

\author{
B I Korelitz, G Y Lauwers, S C Sommers
}

\begin{abstract}
Serial sections of 812 rectal biopsy specimens from 356 Crohn's disease patients were analysed for mucosal epithelial dysplasia. Dysplasia was found in 18 patients $(5 \%)$, with four showing dysplasia on repeat biopsy specimen. In these 22 biopsy specimens the dysplasia was mild in 13, moderate in nine, and severe in none. Subsequently, three patients (17\%) developed neoplasms including carcinoma in two and an adenomatous polyp in one. In colectomy specimens which showed dysplasia, significantly more dysplastic changes were found in seven patients who underwent colonic resection than in $\mathbf{1 0}$ others who underwent operation but had no prior dysplasia $(p<0.001)$. Thirteen patients still have their rectum in situ and remain at risk of developing colonic cancer. Four carcinomas developed in patients with Crohn's disease who did not have dysplasia on rectal biopsy specimen.
\end{abstract}

The increased risk of cancer of the bowel in patients with Crohn's disease has been established. ${ }^{1-5}$ This risk is greater in patients with Crohn's disease of the colon or ileocolitis ${ }^{145}$ than those with ileitis. ${ }^{235}$ Epithelial dysplasia in ulcerative colitis is regarded as a predictor of future colorectal carcinoma or as a sign that it may already coexist, ${ }^{6}$ and has served as the basis for surveillance programmes. ${ }^{7}$ Dysplasia in conjunction with Crohn's disease has also been reported, ${ }^{8-11}$ but the limited data have not yet led to acceptance of regular colonoscopic surveillance in these patients.

The most accessible segment of the intestinal tract for biopsy is the rectum. Influenced by the high yield of epithelial dysplasia found on rectal biopsy specimens in patients with ulcerative colitis, ${ }^{612-16}$ a similar search for dysplasia was undertaken in patients with Crohn's disease to determine its frequency and importance.

\section{Methods}

During the 10 years between 1971 and 1981, 838 sigmoidoscopic rectal biopsies were performed in 356 patients with Crohn's disease in any part of the digestive tract. These biopsies were undertaken for diagnosis, differential diagnosis, to determine the extent of bowel involvement, and for research goals such as response to drug therapy, as well as to identify dysplasia. Microscopic slides of these 838 biopsy specimens were reviewed for dysplasia as well as for any other histological changes in the rectal mucosa. The review was made by a pathologist (SCS) who was blinded as to the clinical severity, duration, or gross extent of the Crohn's disease. Colonic mucosal dysplasia was considered histologically and cytologically to be the same in Crohn's disease as in ulcerative colitis, and the same diagnostic criteria established by the study group for ulcerative colitis were utilised. ${ }^{17}$ One of us (SCS) was a participant in the study group and its publication. The biopsy specimens interpreted as showing dysplasia were entered into a separate blind coded study intermingled with three times as many sets of rectal biopsy specimens from Crohn's disease patients which showed only inflammatory and regenerative changes. These were reviewed by another pathologist (GYL) who also has special experience in the field of dysplasia.

All biopsy specimens had been taken by one of us (BIK) using the bronchoscopic type cupped forceps. ${ }^{18}$ In all instances, three specimens were taken at least $1 \mathrm{~cm}$ apart from the most distal $10 \mathrm{~cm}$ of rectal mucosa. The specimens were fixed in Bouin's solution, processed in the usual manner, and stained with haematoxylinphloxin-safranin trichrome. The overall range of serial sections examined for each specimen was three to 73. Clinical correlations were made with patient age, duration of disease, distribution of disease, gross sigmoidoscopic findings, coincidental histological findings, and the dysplasia. Follow up was then attempted to determine the current clinical status of the patients, evidence of dysplasia in later biopsy specimens, histological evidence of dysplasia in any later resected diseased colonic segment, and the later development of carcinoma.

Low grade dysplasia, comprising slight and moderately severe degrees of dysplasia, was characterised by enlarged, usually hyperchromatic, crowded and stratified crypt nuclei. These nuclei were largely confined to the basal half of the cells. Sometimes incomplete cell maturation or dystrophic goblet cells with altered nuclear polarity were present. High grade dysplasia, comprising severe dysplasia and carcinoma in situ, was characterised by nuclear stratification extending into the upper parts of the cells, with nuclear pleomorphism and loss of polarity. For the purpose of this study, low grade dysplasia was further divided into mild and moderate as described earlier. ${ }^{19}$ Examples of mild and moderate dysplasia are shown in Figures 1 and 2. Indefinite or doubtful instances of dysplasia were excluded from this study. Other histological changes also were identified by previous established criteria. ${ }^{19}$

Microscopic slides of surgical resection specimens were examined field by field of mucosa; each field was about $1.5 \mathrm{~mm}$ in diameter and approximately the same size as the mucosa included in the individual serial sections of a biopsy specimen. Using the criteria noted above, dysplasia, if present, was identified and the number of total fields containing dysplasia were determined.

Comparisons were made between the groups 
Figure 1 (patient 7): Left moderate dysplasia. The nuclei are hyperchromatic, relatively regular with clumping of chromatin and small nucleoli. Overlapping of the nuclei is prominent and largely confined to the lower half of the cells: their polarity is preserved. Mild decrease of goblet cells is present. (Haematoxylin and eosin, original magnification $\times 400$.)

Right - the nuclei are less hyperchromatic and some are vesicular. Stratification is more prominent. Mucin is abnormally present in columnar cells rather than goblet cells. The striking feature here is the atypical pseudocribiforming (see arrows). (Haematoxylin and eosin, original magnification $\times 400$.)
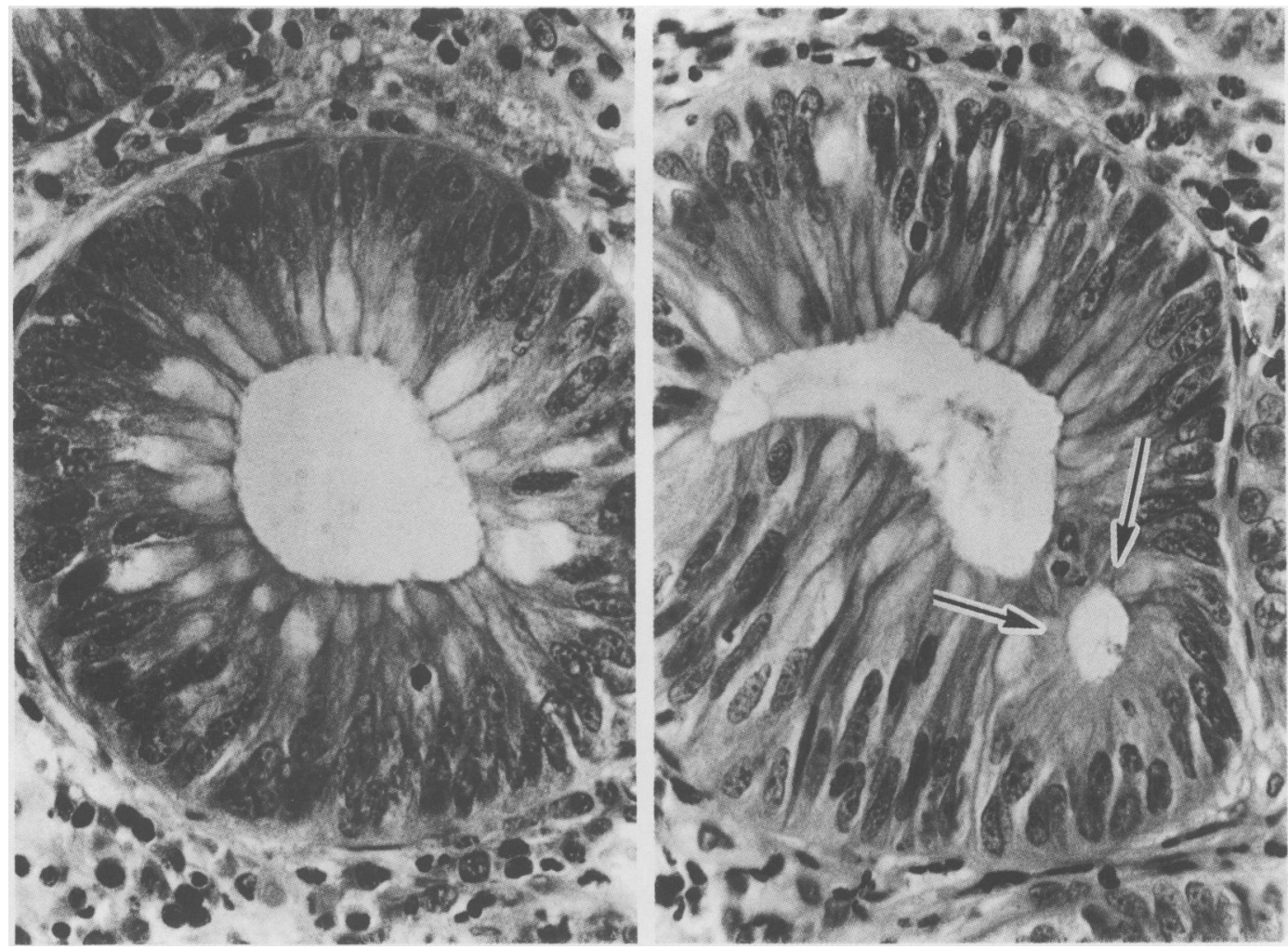

with and without dysplasia in rectal biopsy specimens and dysplasia in specimens of colon later resected for Crohn's disease using the statistical $\chi^{2}$ test. ${ }^{20}$ Also, the number of microscopic fields with dysplasia in resection specimens were compared in the patients with and without dysplasia in biopsy specimens.

\section{Results}

In all, 838 rectal biopsy specimens from 356 patients with Crohn's disease were reviewed histopathologically, and 26 of these were considered insufficient for diagnosis. Dysplasia was recognised by one of us (SCS) in 22 of 812 sets of biopsy specimens belonging to 18 patients $(5 \%)$. In none of the 22 sets was the dysplasia severe; in 13 it was mild and in nine it was moderate. Review of slides by the other pathologist (GYL) resulted in an overall correlation of interpretation of $93 \%$ and a correlation of $75 \%$ with the original determination of low grade dysplasia. Other accompanying histological findings are listed in Table I.

Clinical features in the 18 patients in whom dysplasia was found are presented in Table II.

TABLE I Other histological findings in 812 rectal biopsy specimens of patients with Crohn's disease

\begin{tabular}{lr}
\hline Lesion & No \\
\hline No lesion & 329 \\
Mucosal oedema only & 86 \\
Mucosal fibrosis & 79 \\
Acute and chronic inflammation & 79 \\
Focal or slight chronic inflammation & 72 \\
Moderate chronic inflammation & 50 \\
Oedema with slight chronic inflammation & 24 \\
Oedema with moderate chronic inflammation & 16 \\
Oedema with acute and chronic inflammation & 4 \\
Oedema with focal acute inflammation & 3 \\
Mucosal atrophy & 6 \\
Granulomas & 12 \\
Microgranulomas (includes the 12 with granulomas) & 61
\end{tabular}

Dysplasia was found again on a second occasion in four of these patients (patients 3,6,7, and 12). In one of the four the diverted strictured rectum was later resected (patient 6 ) and it contained no carcinoma. In the other three, subsequent biopsy specimens showed no dysplasia but in one (patient 12), an adenomatous polyp was present within a polypoid cluster at the site of an ileorectal fistula.

Twelve of the 18 patients underwent surgical resection for Crohn's disease at some time after the finding of dysplasia on rectal biopsy specimen. In eight of the 12 the operation was performed at this hospital. Surgical procedures included five ileocolectomies with reanastomosis (patients 3, 5, 8, 10, and 14), one ileocolectomy and abdominoperineal resection (patient 11), one segmental resection of the colon with reanastomosis (patient 18), and the one resection of the diverted rectal segment after earlier ileocolic resection with ileostomy (patient 6). The slides were reviewed in all eight patients. In two of the eight minimal colonic tissue was included in the resected specimen and it showed eight no dysplasia was found in 138 microscopic fields (patient 6), 76 fields (patient 8), and 131 fields (patient 18). In the remaining three, moderate dysplasia was found in five of the 269 microscopic fields (patient 5), nine of the 85 fields (patient 10), and one of 43 fields (patient 11). One of these eight (patient 8) died later of sepsis, and moderate dysplasia was found at necropsy in one of 29 microscopic fields of slides from the residual colon. Carcinoma was not found in any of these eight patients.

Four of the 12 who had surgery underwent ileocolectomy elsewhere, as determined by follow up information (patients 2, 4, 15, and 17). Two of the four are clinically well after total proctocolectomy and ileocolectomy (patients 2 no dysplasia (patients 3 and 14). In three of the 
Figure 2 (patient 10): Left mild dysplasia. The vesicular nuclei, with conspicuous small nucleoli, display some degree of pleomorphism and overlapping. The polarity is somewhat disturbed, some stratification is present.

There is no depleting of mucus: nonetheless a dysplastic goblet cell is identified (see arrow).

(Haematoxylin and eosin original magnification $\times 400$.)

Right - the nuclei are hyperchromatic, but their polarity is correct and almost Some overlapping is present due to the increased number of columnar cells

(Haematoxylin and eosin original magnification $\times 400$.) no stratification is identified.

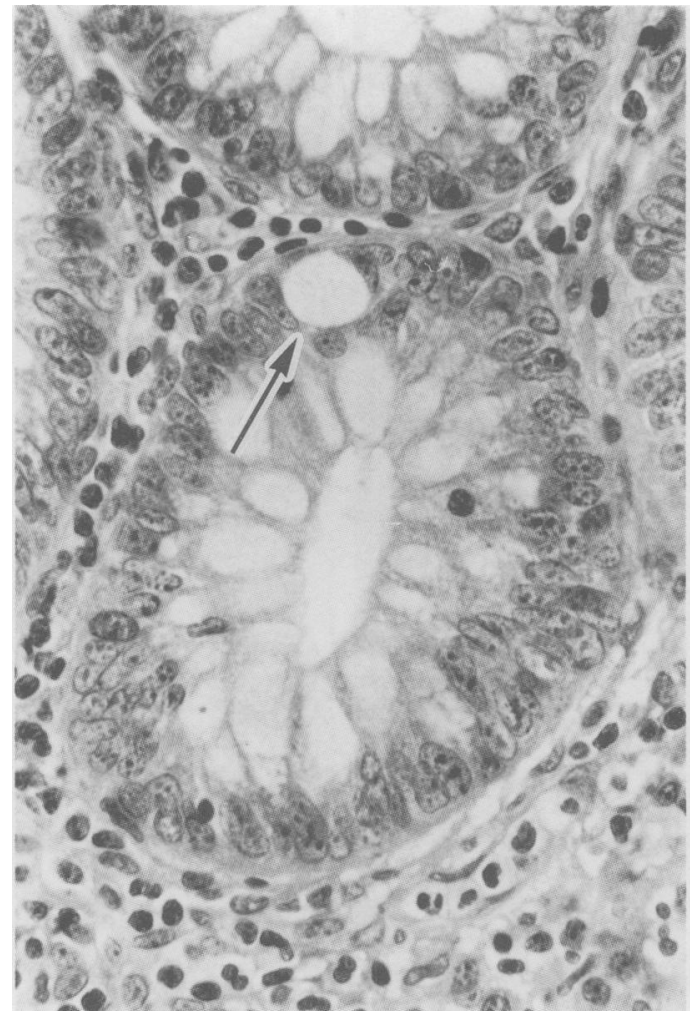

and 15). Slides of the colon were obtained for review in patient 15 and no dysplasia was found in 142 fields.

Of the remaining two patients whose later course was disclosed at follow up, one 21 year old man (patient 4), in whom moderate dysplasia was found on rectal biopsy specimen, had suffered with Crohn's disease for 13 years, had undergone a subtotal colectomy with ileosigmoidostomy, and had very active recurrent disease proximal and distal to the anastomosis complicated by an abdominal wall fistula. He had been lost to follow up for 13 years. Subsequent information showed that he underwent ileoproctectomy for carcinoma of the rectum seven years after the biopsy specimen that showed dysplasia and had subsequently died of metastatic mucinous adenocarcinoma.

The other, a 36 year old woman (patient 17), in whom moderate dysplasia was found on rectal

TABLE II Clinical features in 18 patients with Crohn's disease and rectal mucosal dysplasia

\begin{tabular}{|c|c|c|c|c|c|c|}
\hline \multirow[b]{2}{*}{ Patient } & \multicolumn{2}{|c|}{$\begin{array}{l}\text { Degree of low } \\
\text { grade dysplasia }\end{array}$} & \multirow{2}{*}{$\begin{array}{l}\text { Age at } \\
\text { biopsy } \\
\text { (yrs) }\end{array}$} & \multirow[b]{2}{*}{ Distribution of disease } & \multirow{2}{*}{$\begin{array}{l}\text { Duration } \\
\text { of } \\
\text { disease } \\
\text { (yrs) }\end{array}$} & \multirow{2}{*}{ Appearance } \\
\hline & lst & Later & & & & \\
\hline 1 & $\mathrm{~S}$ & 0 & 17 & Colitis & 3 & Inflammation \\
\hline 2 & $\mathrm{~S}$ & - & 16 & Ileocolitis & 3 & Inflammation \\
\hline 3 & $\mathrm{~S}$ & M & 32 & Postresection ileum \& rectum & 13 & Stricture \\
\hline 4 & $\mathbf{M}$ & 0 & 1 & Postresection ileum \& rectum & 13 & Stricture \\
\hline 5 & $\mathbf{M}$ & 0 & 17 & Colitis & 2 & Inflammation \\
\hline 6 & $\mathrm{~S}$ & $\mathrm{~S}$ & 28 & Postresection diverted rectum & 8 & Stricture \\
\hline 7 & $\mathrm{~S}$ & $\mathbf{M}$ & 40 & Colitis & 10 & Minimal inflammation \\
\hline 8 & $\mathbf{M}$ & 0 & 16 & Extensive & 5 & Normal \\
\hline 9 & $\mathrm{~S}$ & - & 22 & Colitis & 10 & Normal \\
\hline 10 & $\mathrm{~S}$ & 0 & 26 & Extensive & 4 & Normal \\
\hline 11 & $\mathbf{M}$ & 0 & 29 & Postresection ileum \& colon & 9 & $\begin{array}{l}\text { Polypoid rectovaginal } \\
\text { fistula }\end{array}$ \\
\hline 12 & S & S & 62 & Ileitis, ileorectal fistula & 32 & Polypoid \\
\hline 13 & $\mathbf{M}$ & 0 & 49 & Postresection ileitis & 23 & Hypertrophied \\
\hline 14 & $\mathrm{~S}$ & - & 17 & Ileocolitis & 6 & Normal \\
\hline 15 & $\mathbf{M}$ & 0 & 19 & Ileocolitis & 8 & Polypoid \\
\hline 16 & $\mathrm{~S}$ & 0 & 6 & Colitis & 0 & Inflammation, polypoid \\
\hline 17 & $\mathbf{M}$ & 0 & 36 & Colitis & 23 & Pasty \\
\hline 18 & $\mathbf{M}$ & 0 & 18 & Colitis & 3 & Minimal inflammation \\
\hline
\end{tabular}

$\mathbf{M}=$ moderate; $\mathbf{S}=$ slight

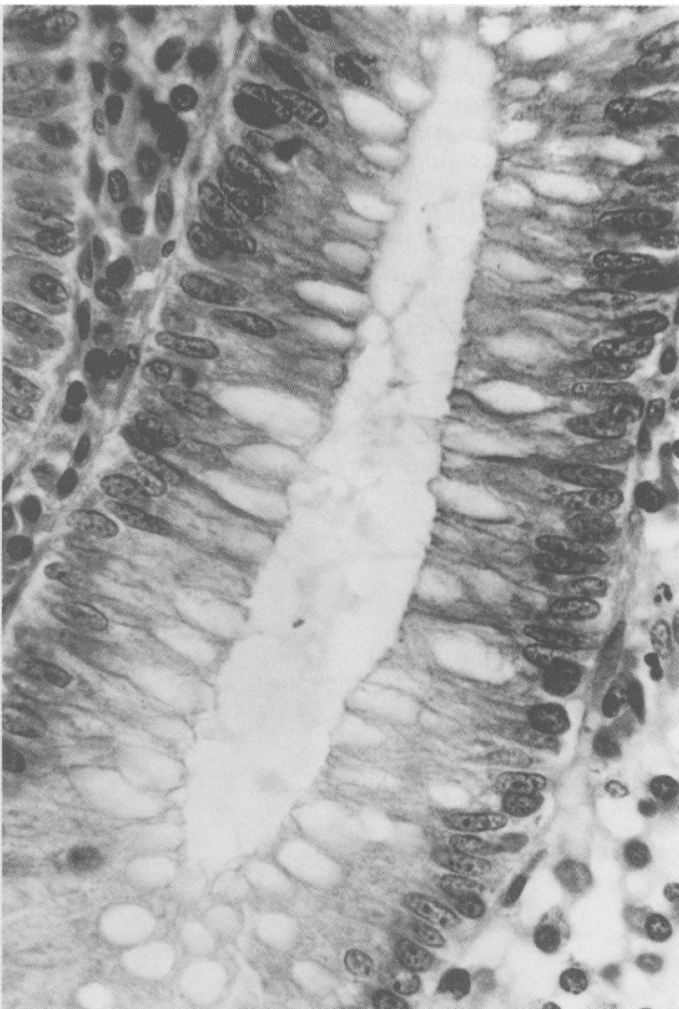

biopsy specimen, had suffered with Crohn's disease for 23 years. Follow up investigation showed that a sigmoidal lesion had been found at colonoscopy and biopsy specimens showed adenocarcinoma. The specimens from and proximal to the lesion also showed moderate dysplasia. A liver biopsy specimen showed adenocarcinoma and she died of metastases one year after dysplasia was found by rectal biopsy specimen.

In 13 patients, the rectum remains in situ (Table III). These 13 patients may develop carcinoma in this segment and at least 10 of them have been followed up with periodic sigmoidoscopy or colonoscopy and biopsies.

Records of patients with widespread Crohn's disease affecting the colon seen in the same private practice were reviewed to find those with rectal biopsy specimens that were negative for dysplasia who later underwent resection. Ten cases were found and the slides of the colon were reviewed. No dysplasia was found in nine patients in a search of 2152 total fields (range 108-461 field each). In the 10th patient, moderate dysplasia was found in three and slight dysplasia in another three of the 115 fields examined. The patient is clinically well with an ileostomy after total proctocolectomy.

Statistical comparisons of the number of patients with or without any colonic dysplasia in the resection specimens in the presence or absence of prior dysplasia in biopsy specimens showed no significant difference between these two groups, using the $\chi^{2}$ test. This could be related to the small numbers involved. The numbers of microscopic fields with dysplasia in the resected colons, however, were highly significantly more frequent in the patients with dysplastic rectal biopsy specimens than in those without, $\chi^{2}=18.9(\mathrm{p}<0.001)$.

During the same 10 year period of this study, 
TABLE III Follow up status of 18 patients with Crohn's disease and rectal mucosal dysplasia

\begin{tabular}{clc}
\hline Patient & Follow up & $\begin{array}{c}\text { Duration } \\
\text { (yrs) }\end{array}$ \\
\hline 1 & Active disease - no surgery & 16 \\
2 & Clinically well - ileoproctocolectomy & 16 \\
3 & Clinically well - ileocolectomy & 15 \\
4 & Died - ileoproctectomy - carcinoma of rectum & 12 \\
5 & Clinically well - ileocolectomy & 13 \\
6 & Clinically well - abdominoperineal resection & 14 \\
7 & Active disease - no surgery & 14 \\
8 & Died - sepsis - earlier ileocolectomy & 8 \\
9 & Active disease - no surgery & 11 \\
10 & Active disease - ileocolectomy & 11 \\
11 & Clinically well - ileocolectomy - diverted colon & 9 \\
12 & Clinically well - adenomatous polyp of rectum & 41 \\
& - no surgery & 8 \\
13 & Clinically well - perirectal abscess & 7 \\
14 & Active disease - ileocolectomy & 7 \\
15 & Clinically well - ileocolectomy - diverted & 13 \\
& rectum & 24 \\
16 & Clinically well - no surgery & 20 \\
17 & Died - carcinoma of colon & \\
18 & Clinically well - left segmental resection with \\
& anastomosis & \\
\hline
\end{tabular}

four additional carcinomas of the colon complicating longstanding Crohn's disease were found without associated dysplasia on rectal biopsy specimen. These included two carcinomas of the rectum found on rectal biopsy specimen. In one of these the specimens were taken from a tight rectal stricture and in the other the colectomy specimen showed multiple synchronous carcinomas of the colon and areas of dysplasia as well. In the third, carcinoma was found on the colonic side of a strictured ileocolic anastomosis. ${ }^{21}$ The fourth patient was a woman with quiescent longstanding Crohn's disease who had a cervical smear positive for adenocarcinoma. Since she had had a hysterectomy, a new colonoscopy was undertaken to find a primary site of adenocarcinoma. Again, the colonic mucosa was unremarkable as were colonic biopsy specimens except for a longstanding rectovaginal fistula which proved to be the cancer site.

\section{Discussion}

In this study, the yield of dysplasia in rectal biopsy specimens of patients with Crohn's disease was $5 \%$ (18 of 356 ). The study did not include colonoscopic biopsy specimens or exclude rectal biopsy specimens in which the general appearance of the rectal mucosa was normal. In ulcerative colitis, the diagnosis of dysplasia is more secure when the biopsy specimen is taken from macroscopically normal mucosa. In six of the 22 specimens of Crohn's disease positive for dysplasia it was found in mucosa that was not grossly inflamed. The problematic diagnosis of dysplasia made in the presence of inflammation has been discussed elsewhere. ${ }^{22}$

The predictive value of dysplasia for the development of neoplasms of the colon in this study is at least $17 \%$. One patient had a tubular adenoma at the site of an ileorectal fistula, suggesting that the fistula might contribute to the development of a neoplasm. The other two were found at follow up to have died of adenocarcinoma, one originally in the rectum and the other in the sigmoid colon. In another four of the 18 patients dysplasia without carcinoma was found later at the time of ileocolic resection or at necropsy.
Crohn's disease is prone to recurrent bursts of activity for a lifetime. Furthermore, after surgical resection, it often extends to the next proximal bowel segment. Despite the lack of enthusiasm for surgery in Crohn's disease because of this phenomenon, surgical resections have been performed for incapacitating complications more often than in ulcerative colitis. It is perhaps because of these resections of the most diseased segments, which are performed relatively early in the course, that the risk of carcinoma has been considered to be less than in ulcerative colitis.

The evidence for dysplasia either preceding or accompanying carcinoma of the bowel in Crohn's disease is well documented. ${ }^{289}$ When dysplasia accompanies the carcinoma, the two may be distant from each other.$^{823}$ Furthermore, the carcinoma may be multifocal in origin. ${ }^{21} 23-25$

Consideration of a surveillance programme when Crohn's disease affects the colon seems warranted, although the reported incidence of dysplasia in Crohn's disease has been less than in ulcerative colitis. In addition to the high resection rate for Crohn's disease, this may be explained by the difficulty in taking biopsy specimens because of narrowed lumens, strictures, and fistulas, which make colonoscopy difficult. The value of taking random rectosigmoidal biopsy specimens has been noted previously in these situations. ${ }^{8}$

Sigmoidoscopy and biopsies in surveillance for dysplasia complicating Crohn's disease should be considered on the same basis as in ulcerative colitis. ${ }^{16}$ The overall yield of dysplasia on rectal biopsy specimens in patients with Crohn's disease in this study is $5 \%$, a figure derived by including patients with normal looking rectal mucosa $(30 \%)$. Since the finding of dysplasia predicts the development or coincidence of neoplasm in $17 \%$, including colorectal carcinoma in at least $11 \%$, the rectal biopsy seems warranted when sigmoidoscopy or colonoscopy is being performed in the course of the normal management of Crohn's disease.

1 Weedon DD, Shorter RG, Ilstrup DM, Huizenga KA, Taylo WT. Crohn's disease and cancer. N Engl F Med 1973; 289: 1099-103.

2 Traube J, Simpson S, Riddell RH, Levin B, Kirsner JB. Crohn's disease and adenocarcinoma of the bowel. Dig Dis Sci 1980; 25: 939-44.

3 Gyde SN, Prior P, MacCartney JC, Thompson H, Waterhouse JAH, Allan RN. Malignancy in Crohn's disease. Gut 1980 21: $1024-9$

4 Greenstein AJ, Sachar DB, Smith H, Janowitz HD, Aufses AH Jr. A comparison of cancer risk in Crohn's disease and ulcerative colitis. Cancer 1981; 48: 2742-5.

5 Korelitz BI. Carcinoma of the intestinal tract in Crohn's disease: Results of a survey conducted by the National Foundation for Ileitis and Colitis. Am $\mathcal{F}$ Gastroenterol 1983 78: 44-6.

6 Morson BC, Pang LSC. Rectal biopsy as an aid to cancer control in ulcerative colitis. Gut 1967; 8: 423-34.

7 Korelitz BI. Inflammatory bowel disease and cancer. In: Berk JE, ed. Bockus gastroenterology. Fourth edition. Phila-

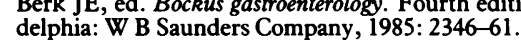

8 Craft CF, Mendelsohn G. Cooper HS, Yardley JH. Colonic 'precancer' in Crohn's disease. Gastroenterology 1981; 80; 578-84

9 Simpson S, Traube J, Riddell RH. The histologic appearance of dysplasia (precarcinomatous change) in Crohn's disease of the small and large intestine. Gastroenterology 1981; 81 492-501.

10 Shamsuddin AKM, Phillips MR. Preneoplastic and neoplastic changes in colonic mucosa in Crohn's disease. Arch Pathol Lab Med 1981; 105: 283-6.

11 Warren R, Barwick KW. Crohn's colitis with carcinoma and dysplasia. Report of a case and review of 100 small and large dysplasia. Am.7 Surg Pathol 1983; 7: 151-9. 
12 Morson BC. Rectal biopsy in inflammatory bowel disease. NEnglf Med 1972; 287: 1337-9.

13 Myrvold HE, Kock NG, Alren C. Rectal biopsy and precancer in ulcerative colitis. Gut 1974; 15: 301-4.

14 Riddell RH, Morson BC. Value of sigmoidoscopy and biopsy in detection of carcinoma and premalignant change in in detection of carcinoma and premative colitis. Gut 1979; 20: 575-80.

15 Nugent FW, Haggett RC, Colcher H, Kutteruf GC. Malignant potential of chronic ulcerative colitis. Gastroenterology 1979; 76: 1-5. 16 Fochios SE, Sommers SC, Korelitz BI. Sigmoidoscopy and
biopsy in surveillance for cancer in ulcerative colitis. $\mathcal{F}$ Clin Gastroenterol 1986; 8: 249-54.

17 Riddell RH, Goldman H, Ransohoff DF, et al. Dysplasia in inflammatory bowel disease: Standard classification with inflammatory bowel disease: Standard classification with provisional clinical application. Hum Pathol 1983; 14:

18 Fochios SE, Korelitz BI. The role of sigmoidoscopy and rectal biopsy in diagnosis and management of inflammatory bowel disease. Am ₹ Gastroenterol 1988; 83: 114-9.

19 Rotterdam H, Sommers SC. Biopsy diagnosis of the digestive tract. New York: Raven Press, 1981: 482.

20 Feinstein AR. Clinical biostatistics. St Louis: Mosby. 1977:

21 Cooper DJ, Weinstein MA, Korelitz BI. Complications of Crohn's disease predisposing to dysplasia and cancer of the intestinal tract: considerations of a surveillance program. $\mathcal{f}$ Clin Gastroenterol 1984; 6: 217-24.

22 Korelitz BI. Response to review of selected summary. Gastroenterology 1986; 92: 2044.

23 Hamilton SR. Colorectal carcinoma in patients with Crohn's disease. Gastroenterology 1985; 89: 398-407.

24 Castellano TJ, Frank MS, Brandt LS, Mahadevia P. Metachronous carcinoma complicating Crohn's disease. Arch
chrono chronous carcinoma complicatin
Intern Med 1981; 141: 1074-5.

- 25 Shorter RG. Risks of intestinal cancer in Crohn's disease. Dis Colon Rectum 1983; 26: 686-9. 